\title{
An IoT-inspired Cloud-based Web Service Architecture for e-Health Applications
}

\author{
Loreto Pescosolido*, Riccardo Berta ${ }^{\dagger}$, Lorenzo Scalise ${ }^{\ddagger}$, Gian Marco Revel ${ }^{\ddagger}$, \\ Alessandro De Gloria ${ }^{\dagger}$, Gianni Orlandi* \\ *DIET, University of Rome "La Sapienza", Rome, Italy. Email: \{loreto.pescosolido,gianni.orlandi\}@uniroma1.it \\ ${ }^{\dagger}$ DITEN, University of Genoa, Genova, Italy, Email: \{ricccardo.berta, alessandro.degloria\}@unige.it \\ ${ }^{\ddagger}$ DIISM, Università Politecnica delle Marche, Ancona, Italy, Email: \{1.scalise, gm.revel\}@univpm.it
}

\begin{abstract}
E-Health services can take advantage of the technological achievements in the area of the Internet of Things (IoT), and of the cost reduction and increasing user-friendliness of health monitoring devices. Homes equipped with environmental sensors, physiological parameters monitoring devices, and home automation devices, could become the "hardware" of an "operating system" for application developers and service providers. The system would expose web services through a unique cloud infrastructure for users' data collection and storage, administration and billing, and healthcare service provisioning applications by possibly multiple third parties. We present an instance of a cloud-based web server which relies on a "home system" for the collection of information from an heterogeneous set of devices, providing a high level description of the proposed overall architectural model, of the induced opportunities from the market perspective, and of how it could be used by healthcare applications developers and service providers, including details on how the web server Application Programming Interfaces (API) is implemented in our instance.
\end{abstract}

Index Terms-e-Health, Internet of Things (IoT), Web of Things (WoT), REST, web services, cloud platforms, s-Health.

\section{INTRODUCTION}

E-Health services use data acquired by heterogeneous home ambient sensors, body (wearable) sensors, personal nonwearable sensors, home automation devices, and medical devices to offer health-related added value services and products. Research in this field has been on the way for more than a decade and the technological achievements in this area make it possible today to deploy heterogeneous types of devices to sense people physiological parameters, human activity (positioning devices, accelerometers) as well as ambient parameters (temperature, pressure, humidity, light, sound, etc.) at a relatively low cost. Sensors and devices are interconnected to form wireless sensor networks which constitute the data source for many research oriented applications and commercial products [1]. In the recent years, the advent of the Internet of Things (IoT) paradigm [2], has been transforming the way systems and services based on wireless sensors and devices are designed and utilized. E-Health-oriented IoT-based applications, thanks to the reduced development and production costs, have the potential to significantly expand the market of health monitoring systems beyond the domain of systems designed to operate in hospitals, healthcare facilities, and clinical laboratories, towards a new domain of systems deployed in the places of everyday life: homes, offices, sporting clubs, etc. The interested reader can check, e.g., [3] for a comprehensive state of the art in this field.
The IoT paradigm can boost the e-Health industry by (i) enabling an easier interconnection of wireless sensors and embedded devices using heterogeneous link layer technologies ${ }^{1}$ through dedicated protocols (e.g., 6LowPan, CoAP, etc.) [5]; and (ii) adopting a service-oriented architecture and leveraging the convergence towards the Web of Things (WoT) [6] opening up to an easier development of new added-value services by healthcare service providers.

This work focuses on the latter of the two above issues, and presents a possible implementation of a Cloud-based serviceoriented architecture which makes it possible to develop different healthcare applications for different types of healthcare service providers utilizing a common infrastructure. We highlight that a modular architecture implemented through REST web services can be directly mapped to new market opportunities and business models, making it simple for different types of stakeholders to enter the market. Today, there already exists a plethora of health-monitoring applications for mobile devices or desktops by different vendors. Each of these apps relies on its own cloud server for users' data storage, and users data are stored and used in a non-necessarily standardized way. Moreover, most of these systems are proprietary and closed to the development of third party apps. The current paradigm results in a replication of the same basic functionalities - users' data collection, storage, and elaboration - which turns out in an overall system inefficiency from several points of view, including privacy, security, and network bandwidth occupation. Furthermore, it is hard for any potential stakeholder to enter the market unless it has the technical and commercial strength to develop and sell a complete vertical solution. In contrast, an open model in which users' data from sensors and medical devices at home are transferred to a unique cloudbased infrastructure, and multiple healthcare service providers can access and elaborate such data through a web service Application Programming Interface (API), could create new market opportunities. There is a direct relationship between the openness of the considered framework and the possibility to let new business opportunities emerge.

\footnotetext{
${ }^{1}$ Link layer technologies for the IoT include technologies for resourceconstrained devices, such as Bluetooth, BLE, IEEE 802.15.4, 6LowPan, as well as technologies for non-constrained devices, such as WiFi and cellular networks [4]. A notable implementation of an IoT/WoT-oriented health monitoring system through a domestic sensor network, though not including a cloud component, as done by us, is proposed in [5].
} 
The paper is organized as follows. In Section II we provide the overall system description and point out several potentially emerging business models for operators in the area of e-Health systems. In Section III we describe more in detail the proposed Cloud-based platform and REST web services. Finally, in Section IV we provide our concluding remarks.

\section{SYSTEM DESCRIPTION AND RELATED BUSINESS} MODELS

The proposed system is composed of the parts represented in Fig. 1 and described at a high level in the following, along with the suggestion of possible business models for potential stakeholders that would play the role of providing one of the system components. A more detailed description of the first component, and particularly of the web services, is provided in Section III.

a) Cloud Platform and web services: This is the system core component. It hosts the users database, which contains data retrieved by the users' home systems (see below), and exposes an API for applications implementing different healthcare services. The Cloud platform also requires a billing system, an alert system, and a web portal through which citizens can have access to their data, check the list of the available healthcare services, or check the home system configuration required to subscribe to a particular healthcare service. The exposed API, in a nutshell, makes the data acquired from end users available to healthcare services which rely on them to implement their healthcare applications. The platform will be designed to interoperate both with the users' home systems (as defined below) or with mobile devices (typically smartphones) able to send data acquired from body sensors when the user is outside the house.

From a business logic point of view, the owner of the platform would typically be a big telecom/internet industry player, possibly operating on the basis of a public tender at a regional or national level. The size and commercial strength of the owner must be adequate to sustain the burden of managing the data of possibly millions of people while satisfying suitable requirements in terms of infrastructure adequateness $^{2}$ (bandwidth, storage, computing capacity, stability, reliability, data integrity, security, and privacy). Having data collected and made available to healthcare service providers fulfilling clear requirements, established by law, in terms of the above mentioned issues, would certainly represent an improvement with respect to the current situation in which such data are collected and used by a plethora of apps with very limited control [7]. The web services operating on top of the cloud infrastructure expose a suitable API which allows dedicated apps developed by third parties to use the data in it for added-value healthcare services (see below). The web services could be developed by a company different from the cloud infrastructure owner. There are two, possibly coexisting, business models for a company that would undertake this

\footnotetext{
${ }^{2}$ In our ongoing experimental validation, whose description is outside the scope of this paper, we will use a cloud infrastructure provided by Telecom Italia, which complies with state-of-the-art standards in terms of security, privacy, stability and reliability.
}

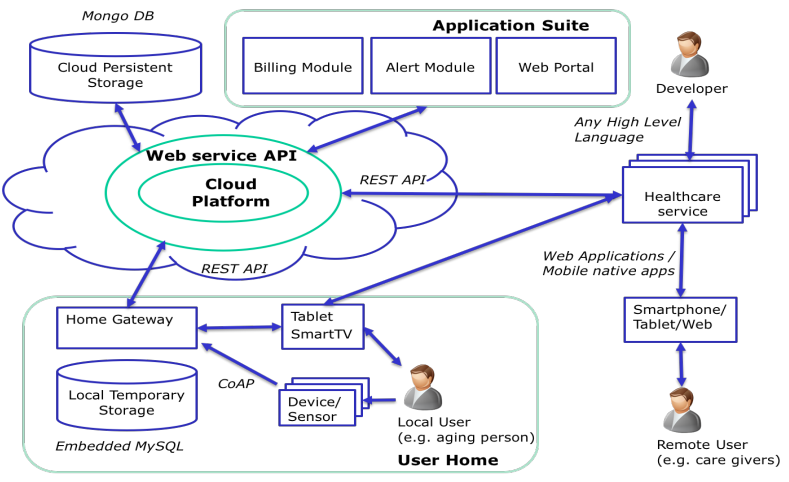

Figure 1. System overview

challenge. One business model would target, as customers, companies acting as healthcare service providers or appdevelopers. The web services would offer the possibility to develop new apps without the need (and the associated costs) of implementing a complete internal data management system. The Cloud platform owner and web service developer would obtain revenues by billing the healthcare service providers for letting them use a suitable subset of the data of the citizens willing to use their apps. The second business model involves the public National Health Service (NHS). There are several objectives that the NHS could achieve in a direct or indirect way. In a direct way, the NHS would be involved by using dedicated web apps to retrieve and present suitably elaborated data about a user's health to healthcare operators. In an indirect way, the NHS would benefit from the effects that a widespread increase of Health self-awareness in citizens would certainly bring in terms of health improvement and healthy lifestyles diffusion, which, in turn, would reduce the public costs of health-related issues. Particularly, it will be possible to achieve: (i) a high degree of de-hospitalization thanks the possibility of performing preliminary medical exams on the basis of individual user's available data, (ii) a reduction of the insurgence of pathologies in a large number of citizens by performing preventive monitoring and issuing of warnings to the users who have subscribed to a health self-monitoring service, (iii) the possibility to conduct cross-sectional as well as longitudinal studies leveraging an an impressive data set, with the possibility to examine the correlation between health data and data provided by other SmartCities IoT applications in use in specific areas, as in the s-Health paradigm [8]. The prospected direct and indirect cost reduction could foster the adoption of a win-win strategy for public authorities and the company/ies providing the cloud-based web service.

b) Healthcare applications and services: The availability of a cloud-based service platform would make it much easier than it is today to develop healthcare applications and services exploiting the rich health-related data set coming from end users' devices. For instance, healthcare services would rely on the web service for a common, unique user authentication. The service developer could ask for a specific API to be implemented by the web service to compute new indicators, high level synthetic parameters, correlation coefficients, and so on, within the cloud (see the definition of the Domain features 
in Section III), without the need of computing them on its own machines. Alternatively, it may just retrieve raw data from the cloud and elaborate them on its own machines. In this regard, our proposed cloud platform is flexible enough to interoperate with both kind of applications. The processed data would then be presented by the application to the clinical partner (e.g., a doctor, or a physiotherapist) for monitoring or diagnosis. As an example, we are currently working on an application for monitoring the state of the hearth and circulatory system functionality of patients and athletes or sportsmen, e.g. to prevent sudden death from cardiac causes, a pathology causing as much as 350000 deceases per year in the U.S. [9].

The market of healthcare service providers would be considerably innovated by the proposed paradigm, since the investments needed to develop an applications would be reduced, making it easier for newcomers to enter the market. The healthcare service would be listed by the web portal (see below) among the services tailored for a particular type of end user, e.g. those affected by a given pathology. In this light, the service provider would benefit of an advertising channel. Besides the web portal (described later) and a billing module, one of the modules of the cloud platform is the alert module: this module could execute checks on data (for service providers and users) and on the devices (for end users and device vendors). The former type of alert would be used by a healthcare service to raise alarms to users and doctors about the insurgence of a possibly critical situation, the latter would inform the user or the vendor about a malfunctioning device.

c) Home System: This is typically composed of a central controller which performs data collection from sensors and medical devices used by citizens to monitor their health and works as a home gateway. The controller would interface with heterogeneous devices such as: (i) physiological parameters monitoring devices (blood pressure monitoring devices and holters, ECG holters, scales, oximeters, saturimeters, glycemia meters, etc.), (ii) devices aimed at detecting the activity of end users (presence detectors, accelerometers, sleep quality monitoring devices, etc.), and (iii) ambient sensors to detect physical parameters (temperature, light, sound level, air pressure, etc..) and home automation devices. Data retrieved from the devices can be stored in a local database and, at least for non time-critical applications, be asynchronously sent to the cloud. We provide technical details of a possible implementation of a Home System which will be integrated with our platform in [10].

From the market perspective, a device producer would have the opportunity to register its devices to the platform, provided that communication protocol in use is compatible with those foreseen for the home system. The device would be listed by the platform web portal (see below) among those that are suitable to be part of the equipment required by a given healthcare service, so that a user can decide to buy it. We mentioned, earlier in this section, that the cloud platform should be flexible enough to receive data from both the home system and from users' smartphones. It is worth pointing out that, from an architectural point of view, the benefit of having a home system performing data collection within the house, instead of relying on a mobile device acting as a data sink even in the house, is that in this way the impact in terms of battery consumption of the smartphones, overall EM footprint, and bandwidth occupation would be considerably reduced, since the communication between the central controller and the cloud would typically use wired connections.

d) Web Portal: The web portal would be the front end of the overall system for the users. In the web portal, a registered user could visualize the available services and the device it should purchase to be sure it can make a given service operational for her/him. Users could also check their administrative position with respect to the distinct healthcare services. A device producer could access ad dedicated area to initiate the procedure to register its devices. Additionally, developer tools may also be accessible through the web portal for service providers to develop their apps.

\section{Cloud-BASEd RESTful WEB SERVICE}

We have implemented an instance of the above described cloud-based web services. The system interface has been designed as an open API to allow developers to build new services leveraging data captured from home and personal devices. The main objective is to lower the barrier between the low level implementation details of the devices and the provision of high-level functionalities. To this end, we have designed a set of open web services following the REST approach [11] at level 3 of the Richardson Maturity Model [12]. The services interfaces are based only on resources identified by a unique Uniform Resource Identifier (URI). All resources are accessed exploiting the semantics of the standard HTTP methods, without inventing other verbs (e.g. GET to access a resource representation, POST to create a new resource, and PUT to modify an existent resource). Moreover, to further facilitate the API adoption, we implement the notion of "hypermedia as an engine of application states" (HATEOAS principle [11]), which is the way the REST approach associates resources with other resources (hypermedia controls). As an example, the representation of a "user" resource contains hyperlinks to the "measurement" resources performed on the "user" by a "device". This approach allows the developer to control the navigation between the REST resources and to understand, in a natural way, the allowed CRUD (Create, Read, Update and Delete) operations. The decision of using resources, HTTP verbs and links (instead of classical remote methods invocations) to provide API access to the e-Health contents is not enough to facilitate developers in understanding the behavior of the e-Health platform. In the design of the resources, we followed a Domain Driven Design approach [13] in order to produce an "Ubiquitous Language": a common and shared language between service developers and device producers. We have developed a Domain Model, involving domain and business experts. The results (resource names in the ubiquitous language) are showed in Table I.

To simplify the usage of the API with different platforms and programming languages, the resources are described using the JavaScript Object Notation (JSON), a common data format 
Table I

PRINCIPAL RESOURCES EXPOSED BY THE WEB SERVICE API

\begin{tabular}{c|l} 
Resource & \multicolumn{1}{c}{ Description } \\
\hline Provider & $\begin{array}{l}\text { An entity (e.g. companies, public bodies, etc.) } \\
\text { who publishes services on the e-Health system. }\end{array}$ \\
\hline Service & $\begin{array}{l}\text { A service offered to the end-users by a provider } \\
\text { through the e-Health system. }\end{array}$ \\
\hline User & $\begin{array}{l}\text { An end-user of the services offered on the e- } \\
\text { Health system by providers }\end{array}$ \\
\hline Device & $\begin{array}{l}\text { A device (environmental sensors, physiological } \\
\text { parameters monitoring devices, domotic appli- } \\
\text { ances, etc.) which provides data about a user or } \\
\text { the environment. }\end{array}$ \\
\hline Measurement & $\begin{array}{l}\text { A physical dimension that can be measured } \\
\text { by a device (e.g. the heart rate, the ambient } \\
\text { temperature, etc.) }\end{array}$ \\
\hline Trigger & $\begin{array}{l}\text { A value measured by a device for a specific user, } \\
\text { stored in the cloud and accessible by services to } \\
\text { implement its behaviors. }\end{array}$ \\
\hline $\begin{array}{l}\text { A description of a set of conditions (e.g. a partic- } \\
\text { ular range of values for a measurement on a user) } \\
\text { of interest for a subset of services. The e-Health } \\
\text { system will inform the services subscribed to the } \\
\text { trigger when conditions become true. }\end{array}$
\end{tabular}

for implementing REST API integration. Here below we provide an example of a JSON representation of an API resource, specifically, a device object, in which the "features" attribute (see below the definition of "features") makes a reference to a feature resource through a URI:

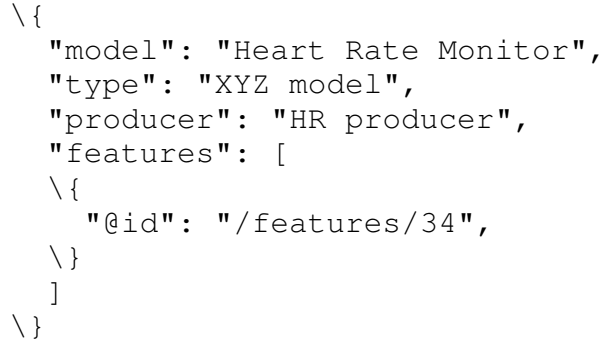

We highlight that the attribute "@id" adds semantic meaning (exploiting a feature of JSON-LD, the JSON extension for linked data [14]) of hyperlink to the "feature", to distinguish its expected type from a plain text field. The link of semantic information with resources properties increases the potential interoperability between the e-Health API and its services.

An important concept of our Domain Model is the notion of "feature". This concept acts as the connection between the IoT world (composed of home appliances and personal devices) and the healthcare applications and services. The platform supports a hierarchical pattern for the definition of features. At the lowest level, there are the base features, which directly map physical parameters acquired by domestic appliances (e.g. ambient temperature) and personal devices (e.g. heart rate variability). At higher levels, we allow the definition of "service oriented features", which are the result of aggregation, post-processing, correlation of the measurements already stored in the cloud in the form of base features. As an example, the feature "physiological stress" can be defined as the time derivative of the "heart rate variability" (which is a base feature). This pattern allows application developers to become data sources themselves, as they could store on the cloud information derived from their internal model. These high-level features (extrapolated from raw data with advanced algorithms, like deep learning) could also aggregate and fuse data coming from other SmartCities infrastructures (e.g., urban sensing ones) and can be shared to other application developers against a use license, enabling a further potentially disruptive market channel.

\section{CONCLUSION}

Cloud-based web service-oriented architectures can expand the market of e-Health, m-Health, and s-Health applications by making it easier for stakeholders to operate in the market or even by introducing new business models. We have described an instance of the proposed cloud-based web service platform, implemented by us, which includes the core elements of the REST service platform and many features required for the healthcare services to operate. We have highlighted the potential of an impressive innovation that could affect the e-Health and s-Health market thanks to the possibility to aggregate and fuse a massive amount of heterogeneous data made available in a secure, stable, and reliable way by the web service operating on top of the cloud infrastructure.

\section{ACKNOWLEDGEMENTS}

This work has been funded by the Italian Ministry of Education, University and Research, under grant no. SCN_00558

- Italian National Smart-Cities project Health@Home.

\section{REFERENCES}

[1] J. Ko, C. Lu, M. B. Srivastava, J. A. Stankovic, A. Terzis, and M. Welsh, "Wireless sensor networks for healthcare," Proceedings of the IEEE, vol. 98, no. 11, pp. 1947-1960, 2010.

[2] J. A. Stankovic, "Research directions for the internet of things," Internet of Things Journal, IEEE, vol. 1, no. 1, pp. 3-9, Feb. 2014

[3] S. R. Islam, D. Kwak, M. H. Kabir, M. Hossain, and K.-S. Kwak, "The internet of things for health care: a comprehensive survey," IEEE Access, vol. 3, pp. 678-708, 2015.

[4] A. Zanella, N. Bui, A. Castellani, L. Vangelista, and M. Zorzi, "Internet of things for smart cities," IEEE Internet of Things Journal, vol. 1, no. 1, pp. 22-32, Feb. 2014.

[5] L. Catarinucci et al., "An iot-aware architecture for smart healthcare systems," Internet of Things Journal, IEEE, vol. 2, no. 6, pp. 515-526, Dec. 2015.

[6] L. Mainetti, V. Mighali, and L. Patrono, "A software architecture enabling the web of things," IEEE Internet of Things Journal, vol. 2, no. 6, pp. 445-454, Dec. 2015.

[7] European Commission, "Green paper on mobile Health ("mHealth")," Apr. 2014. [Online]. Available: http://ec.europa.eu/newsroom/dae/ document.cfm?doc_id=5147.

[8] A. Solanas et al., "Smart health: a context-aware health paradigm within smart cities," Communications Magazine, IEEE, vol. 52, no. 8, pp. 74 81, 2014.

[9] R. R. Liberthson, "Sudden death from cardiac causes in children and young adults," New England Journal of Medicine, vol. 334, no. 16, pp. 1039-1044, 1996.

[10] L. Scalise, F. Pietroni, S. Casaccia, G. M. Revel, A. Monteriù, M. Prist, S. Longhi, and L. Pescosolido, "Implementation of an "at-home" ehealth system using heterogeneous devices," in Proc. 2nd IEEE International Smart Cities Conference (ISC2 2016), Trento, Italy, Sep. 12-15, 2016.

[11] T. Steiner and J. Algermissen, "Fulfilling the hypermedia constraint via http options, the http vocabulary in rdf, and link headers," in Proceedings of the second international workshop on RESTful design. ACM, 2011.

[12] L. Richardson, M. Amundsen, and S. Ruby, RESTful Web APIs. O'Reilly Media, Inc.", 2013.

[13] E. Evans, Domain-driven design: tackling complexity in the heart of software. Addison-Wesley Professional, 2004.

[14] M. Sporny, D. Longley, G. Kellogg, M. Lanthaler, and N. Lindström, "Json-ld 1.0," W3C Recommendation (January 16, 2014), 2014. 